Proc. XII Int. School on Theoretical Physics — Symmetry and Structural Properties of Condensed Matter

\title{
Quantum Filtering Equation for System Driven by Field in a Mixture of Vacuum and Coherent State
}

\author{
A. DĄBROWSKA*
}

Nicolaus Copernicus University in Toruń, Collegium Medicum Bydgoszcz, Jagiellońska 15, 85-067 Bydgoszcz, Poland

\begin{abstract}
We consider the problem of optimal estimation of a state of a quantum system interacting with the Bose field being in a mixture of the vacuum and coherent state. The system and electromagnetic field are described by making use of quantum stochastic unitary evolution. We derive stochastic master equation using Gardiner and Collet's input-output theory and the concept of cascade systems. To find the stochastic evolution of the system conditioned on the result of the quadrature measurement of the output field, we extend the compound system by an ancilla driven by the vacuum and generating the desired non-classical state of light.
\end{abstract}

DOI: 10.12693/APhysPolA.132.112

PACS/topics: quantum measurements, quantum filtration, quantum stochastic calculus of Itô type

\section{Introduction}

The concept of optimal quantum state estimation in the framework of quantum stochastic calculus was developed by Belavkin, Barchielli and their co-workers [1, 2]. In the model the Bose field disturbs the free evolution of a quantum system, but it also enables an indirect and continuous in time observation of the quantum system. The filtering equation describes the evolution of the quantum system conditioned on the results of the continuous in time measurement of the output Bose field (the field after interaction with the system). The filtering theory provides us with the mathematical tools which allow to study the problem of optimal excitation of quantum systems by wave packet traveling in space as well as the problem of storing and retrieving information in quantum systems in an efficient way. Derivation of the filtering equation for the field in the vacuum state one can find, for instance, in [1] and for the squeezed Gaussian state in [3]. The filtering for non-Gaussian states such as continuous-mode single photon state or continuous-mode cat states was studied, for instance, in [4].

In the paper we present derivation of the filtering equation for the Bose field taken in a mixture of vacuum and coherent state using the concept of enlarging the system by ancilla being a source of non-classical signal. It should be stressed that our model of signal generator is the only theoretical tool which serves for derivation of the filtering equations for the non-classical light.

\section{Input-output model of filtering}

Let us consider a quantum system $($ system $\mathcal{S}$ ) interacting with the Bose field [5]. We assume that the unitary operator, $U_{t}$, describing the evolution of the whole system (system $\mathcal{S}$ plus the Bose field) satisfies the Itô quantum stochastic differential equation (QSDE) $[2,6]$ :

$$
\begin{aligned}
\mathrm{d} U_{t} & =\left[L_{S} \mathrm{~d} B_{t}^{\dagger}-L_{S}^{\dagger} \mathrm{d} B_{t}-\left(\frac{\mathrm{i}}{\hbar} H_{S}+\frac{1}{2} L_{S}^{\dagger} L_{S}\right) \mathrm{d} t\right] U_{t}, \\
U_{t=0} & =I,
\end{aligned}
$$

where $L_{S}$ is a coupling operator acting in the Hilbert space of $\mathcal{S}$ and $H_{S}$ is the Hamiltonian of $\mathcal{S}$. Henceforth, we put $\hbar=1$. The Hilbert space of the Bose field is symmetric Fock space having a continuous tensor product structure i.e. $\mathfrak{h}=\mathfrak{h}_{[0, t)} \otimes \mathfrak{h}_{[t,+\infty)}[2,6]$. The operator $U_{t}$ acts trivially in $\mathfrak{h}_{[t,+\infty)}$ and $\left[U_{t}, \mathrm{~d} B_{t}\right]=\left[U_{t}, \mathrm{~d} B_{t}^{\dagger}\right]=0$. Continuous-mode coherent state in $\mathfrak{h}$ is defined as:

$$
|\alpha\rangle=\overrightarrow{\mathrm{T}} \exp \left\{\int_{0}^{+\infty} \alpha(t) \mathrm{d} B_{t}^{\dagger}-\alpha(t)^{*} \mathrm{~d} B_{t}\right\}|v a c\rangle,
$$

where $\overrightarrow{\mathrm{T}}$ stands for the chronological ordering operator and $|v a c\rangle$ is the vacuum state. Note that

$$
\begin{gathered}
\overrightarrow{\mathrm{T}} \exp \left\{\int_{0}^{t} \alpha(s) \mathrm{d} B_{s}^{\dagger}-\alpha(s)^{*} \mathrm{~d} B_{s}\right\}|v a c\rangle= \\
\left|\alpha_{[0, t)}\right\rangle \otimes\left|v a c_{[t, \infty)}\right\rangle,
\end{gathered}
$$

so the continuous-mode coherent state has the factorization property

$$
|\alpha\rangle=\left|\alpha_{[0, t)}\right\rangle \otimes\left|\alpha_{[t,+\infty)}\right\rangle
$$

The mean values of the increments $\mathrm{d} B_{t}, \mathrm{~d} B_{t}^{\dagger}$ and their products for the coherent state are

$$
\begin{gathered}
\left\langle\alpha \mid \mathrm{d} B_{t} \alpha\right\rangle=\alpha(t) \mathrm{d} t, \quad\left\langle\alpha \mid \mathrm{d} B_{t} \mathrm{~d} B_{t}^{\dagger} \alpha\right\rangle=\mathrm{d} t, \\
\left\langle\alpha \mid \mathrm{d} B_{t}^{\dagger} \mathrm{d} B_{t} \alpha\right\rangle=0 .
\end{gathered}
$$

The operators $B_{t}, B_{t}^{\dagger}$ refer to the field before interaction with $\mathcal{S}$ and they are called the input processes, whereas $B_{t}^{\text {out }}=U_{t}^{\dagger} B_{t} U_{t}, B_{t}^{\text {out }}=U_{t}^{\dagger} B_{t}^{\dagger} U_{t}$ describe the field after the interaction with $\mathcal{S}$, and they are called the output processes $[5,6]$. 
3. Reduced dynamics of quantum system coupled to the Bose field in a mixture of vacuum and coherent state

If the Bose field is taken in the state

$$
\rho_{\text {field }}=p|v a c\rangle\langle v a c|+(1-p)| \alpha\rangle\langle\alpha|
$$

with $p \in(0,1]$, then the reduced dynamics of the system $\mathcal{S}$ is given by the density operator [2]:

$$
\rho(t)=p \rho^{00}(t)+(1-p) \rho^{11}(t),
$$

where the matrices $\rho^{00}(t)$ and $\rho^{11}(t)$ satisfy the differential equations

$$
\begin{aligned}
& \dot{\rho}^{00}(t)=-\mathrm{i}\left[H_{S}, \rho^{00}(t)\right]+L_{S} \rho^{00}(t) L_{S}^{\dagger} \\
& -\frac{1}{2}\left\{L_{S}^{\dagger} L_{S}, \rho^{00}(t)\right\}, \\
& \dot{\rho}^{11}(t)=-\mathrm{i}\left[H_{S}, \rho^{11}(t)\right]+L_{S} \rho^{11}(t) L_{S}^{\dagger} \\
& \quad-\frac{1}{2}\left\{L_{S}^{\dagger} L_{S}, \rho^{11}(t)\right\}+\alpha(t)\left[\rho^{11}(t), L_{S}^{\dagger}\right] \\
& +\alpha^{*}(t)\left[L_{S}, \rho^{11}(t)\right]
\end{aligned}
$$

with the initial conditions: $\rho^{00}(0)=\rho^{11}(0)=\rho(0)$.

\section{Model of generation of the Bose field in a mixture of vacuum and coherent state}

As a generator of the field in the mixture of the vacuum and coherent state we consider a two-level system which interacts with the Bose field in the vacuum state and we assume that the evolution of the compound system (the two-level system plus the Bose field) is given by the unitary operator, $\tilde{U}_{t}$ satisfying the equation

$$
\begin{aligned}
& \mathrm{d} \tilde{U}_{t}=\left(L_{A} \mathrm{~d} B_{t}^{\dagger}-L_{A}^{\dagger} \mathrm{d} B_{t}-\frac{1}{2} L_{A}^{\dagger} L_{A} \mathrm{~d} t\right) \tilde{U}_{t}, \\
& \tilde{U}_{t=0}=I,
\end{aligned}
$$

where the coupling operator has the form

$$
L_{A}=\alpha(t)|1\rangle\langle 1| \text {. }
$$

We indicate the upper and ground states of the two-level system, respectively, by $|1\rangle$ and $|0\rangle$. Now one can check that if the initial state of the compound system is given as

$$
\left|\psi_{0}\right\rangle=\left(c_{0}|0\rangle+c_{1}|1\rangle\right) \otimes|v a c\rangle
$$

then the compound system evolves according to the formula

$$
U_{t}\left|\psi_{0}\right\rangle=c_{0}|0\rangle \otimes|v a c\rangle+c_{1}|1\rangle \otimes\left|\alpha_{[0, t)}\right\rangle \otimes\left|v a c_{[t,+\infty)}\right\rangle .
$$

In the limit $t \rightarrow+\infty$, we obtain

$$
\lim _{t \rightarrow+\infty} U_{t}\left|\psi_{0}\right\rangle=c_{0}|0\rangle \otimes|v a c\rangle+c_{1}|1\rangle \otimes|\alpha\rangle .
$$

Thus the Bose field after interaction with the ancilla up to the time $t$ (the output field) is in the state

$$
\varrho_{\text {field }}^{\text {out }}(t)=\left|c_{0}\right|^{2}\left|v a c_{[0, t)}\right\rangle\left\langle\left.\operatorname{vac}_{[0, t)}|+| c_{1}\right|^{2} \mid \alpha_{[0, t)}\right\rangle\left\langle\alpha_{[0, t)}\right| .
$$

\section{Master equation for cascaded system}

We consider the cascaded system consisting of the ancilla (the two-level system) and the system $\mathcal{S}$. We assume that the cascaded system is driven by the Bose field in the vacuum state. The idea of cascaded system was described, for instance, in [5]. When we omit a time shift due to a travelling between the ancilla and $\mathcal{S}$, we get the master equation for the extended system (the system $\mathcal{S}$ plus the ancilla) of the form

$$
\begin{aligned}
& \dot{\tilde{\rho}}(t)=\mathcal{L}_{S} \tilde{\rho}(t)+\mathcal{L}_{A} \tilde{\rho}(t)+\left[L_{A} \tilde{\rho}(t), L_{S}^{\dagger}\right] \\
& +\left[L_{S}, \tilde{\rho}(t) L_{A}^{\dagger}\right]
\end{aligned}
$$

where

$$
\mathcal{L}_{S} \tilde{\rho}=-i\left[H_{S}, \tilde{\rho}\right]+L_{S} \tilde{\rho} L_{S}^{\dagger}-\frac{1}{2}\left\{L_{S}^{\dagger} L_{S}, \tilde{\rho}\right\}
$$

and

$$
\mathcal{L}_{A} \tilde{\rho}=L_{A} \tilde{\rho} L_{A}^{\dagger}-\frac{1}{2}\left\{L_{A}^{\dagger} L_{A}, \tilde{\rho}\right\} .
$$

We assume that initially the extended system is in the state

$$
\tilde{\rho}(t=0)=\rho(0) \otimes \rho_{A}(0),
$$

where

$$
\begin{aligned}
& \rho_{A}(0)=\left|c_{0}\right|^{2}|0\rangle\left\langle 0\left|+c_{0} c_{1}^{*}\right| 0\right\rangle\left\langle 1\left|+c_{0}^{*} c_{1}\right| 1\right\rangle\langle 0| \\
& \quad+\left|c_{1}\right|^{2}|1\rangle\langle 1| .
\end{aligned}
$$

Now by taking the partial trace over the Hilbert space of ancilla, $\mathfrak{h}_{A}$, we get from $(2)$ the equation

$$
\begin{aligned}
& \dot{\tilde{\rho}}_{S}(t)=\mathcal{L}_{S} \tilde{\rho}_{S}(t)+\left[\operatorname{Tr}_{\mathfrak{h}_{A}}\left(L_{A} \tilde{\rho}(t)\right), L_{S}^{\dagger}\right] \\
& +\left[L_{S}, \operatorname{Tr}_{\mathfrak{h}_{A}}\left(\tilde{\rho}(t) L_{A}^{\dagger}\right)\right]
\end{aligned}
$$

for the density matrix

$$
\tilde{\rho}_{S}(t)=\operatorname{Tr}_{\mathfrak{h}_{A}} \tilde{\rho}(t)
$$

describing the reduced dynamics of $\mathcal{S}$. One can check that for the coupling (1) and the initial state (3), we obtain from (4) the set of two differential equations of the form

$$
\begin{aligned}
& \dot{\tilde{\rho}}_{S}^{00}(t)=\mathcal{L}_{S} \tilde{\rho}_{S}^{00}(t) \\
& \dot{\tilde{\rho}}_{S}^{11}(t)=\mathcal{L}_{S} \tilde{\rho}_{S}^{11}(t)+\alpha(t)\left[\tilde{\rho}_{S}^{11}(t), L_{S}^{\dagger}\right] \\
& \quad+\alpha^{*}(t)\left[L_{S}, \tilde{\rho}_{S}^{11}(t)\right]
\end{aligned}
$$

for the matrices $\tilde{\rho}_{S}^{00}(t)=\langle 0|\tilde{\rho}(t)| 0\rangle$ and $\tilde{\rho}_{S}^{11}(t)=$ $\langle 1|\tilde{\rho}(t)| 1\rangle$. Initially we have $\tilde{\rho}_{S}^{00}(0)=\left|c_{0}\right|^{2} \rho(0), \tilde{\rho}_{S}^{11}(0)=$ $\left|c_{1}\right|^{2} \rho(0)$ and

$$
\tilde{\rho}_{S}(t)=\tilde{\rho}_{S}^{00}(t)+\tilde{\rho}_{S}^{11}(t) .
$$

Let us notice that if $\left|c_{0}\right|^{2}=p$ and $\left|c_{1}\right|^{2}=1-p$ this procedure gives the same reduced evolution of $\mathcal{S}$ as before when we considered $\mathcal{S}$ interacting with the Bose field prepared in the mixture of the vacuum and coherent state.

\section{Trajectories for the system driven by the field generated by ancilla}

The quantum filtering equation for a system coupled to the Bose field in the vacuum state is well known and we can write it easily for our extended system consisting of ancilla and $\mathcal{S}$. The filtering equation for the extended system and for the quadrature measurement of the output field has the form 


$$
\begin{aligned}
& \mathrm{d} \hat{\rho}(t)= \\
& \left\{\mathcal{L}_{S} \hat{\rho}(t)+\mathcal{L}_{A} \hat{\rho}(t)+\left[L_{A} \hat{\rho}(t), L_{S}^{\dagger}\right]+\left[L_{S}, \hat{\rho}(t) L_{A}^{\dagger}\right]\right\} \mathrm{d} t \\
& +\left\{\left(L_{S}+L_{A}\right) \hat{\rho}(t)+\hat{\rho}(t)\left(L_{S}^{\dagger}+L_{A}^{\dagger}\right)-K_{t} \hat{\rho}(t)\right\} \mathrm{d} W(t),
\end{aligned}
$$

where $K_{t}=\operatorname{Tr}\left[\left(L_{S}+L_{S}^{\dagger}+L_{A}+L_{A}^{\dagger}\right) \hat{\rho}(t)\right], \mathrm{d} W(t)=$ $\mathrm{d} Y(t)-K_{t} \mathrm{~d} t$ is the innovation Wiener process, and $\mathrm{d} Y(t)=\mathrm{d} B_{t}^{\text {out }}+\mathrm{d} B_{t}^{\text {out } \dagger}$. Note that averaging our filter over all possible trajectories $(\langle\mathrm{d} W\rangle=0)$, we obtain the dynamics given by Eq. (2). Now by taking the partial trace over the Hilbert space of ancilla, we obtain the filter for the system $\mathcal{S}$. Thus the conditional state of $\mathcal{S}$ is given as

$$
\hat{\rho}_{S}(t)=\hat{\rho}_{S}^{00}(t)+\hat{\rho}_{S}^{11}(t),
$$

where

$$
\hat{\rho}_{S}^{00}(t)=\langle 0|\hat{\rho}(t)| 0\rangle, \hat{\rho}_{S}^{11}(t)=\langle 1|\hat{\rho}(t)| 1\rangle .
$$

One can check that the matrices $\hat{\rho}_{S}^{00}(t), \hat{\rho}_{S}^{11}(t)$ satisfy the stochastic differential equations

$$
\begin{aligned}
& \mathrm{d} \hat{\rho}_{S}^{00}(t)=\mathcal{L}_{S} \hat{\rho}_{S}^{00}(t) \mathrm{d} t \\
& \quad+\left\{L_{S} \hat{\rho}_{S}^{00}(t)+\hat{\rho}_{S}^{00}(t) L_{S}^{\dagger}-K_{t} \hat{\rho}_{S}^{00}(t)\right\} \mathrm{d} W, \\
& \mathrm{~d} \hat{\rho}_{S}^{11}(t)=\left\{\mathcal{L}_{S} \hat{\rho}_{S}^{11}(t)+\alpha(t)\left[\hat{\rho}_{S}^{11}(t), L_{S}^{\dagger}\right]\right. \\
& \left.\quad+\alpha^{*}(t)\left[L_{S}, \hat{\rho}_{S}^{11}(t)\right]\right\} \mathrm{d} t \\
& \quad+\left\{L_{S} \hat{\rho}_{S}^{11}(t)+\hat{\rho}_{S}^{11}(t) L_{S}^{\dagger}\right. \\
& \left.\quad+\hat{\rho}_{S}^{11}(t)\left(\alpha(t)+\alpha^{*}(t)\right)-K_{t} \hat{\rho}_{S}^{11}(t)\right\} \mathrm{d} W,
\end{aligned}
$$

with $\hat{\rho}_{S}^{00}(0)=\left|c_{0}\right|^{2} \rho(0), \hat{\rho}_{S}^{11}(0)=\left|c_{1}\right|^{2} \rho(0)$.

\section{Conclusions}

Our solution is equivalent to the filter derived in [4] and it can be easily generalised to the case of a mixture of any number of coherent states. The main restriction of our model is the fact that we cannot consider as an input a superposition of coherent states because the ancilla is not a source of such states. One can find the filtering for the counting process using the same procedure.

\section{Acknowledgments}

This paper was partially supported by the National Science Center project 2015/17/B/ST2/02026.

\section{References}

[1] A. Barchielli, V.P. Belavkin, J. Phys. A Math. Gen. 24, 1495 (1991).

[2] A. Barchielli, in: Lecture Notes Math. 1882, Eds. S. Attal, A. Joye, C.-A. Pillet, Springer, Berlin 2006, p. 207.

[3] A. Dąbrowska, J.E. Gough, Russ. J. Math. Phys. 23, 172 (2016).

[4] J.E. Gough, M.R. James, H.I. Nurdin, J. Combes, Phys. Rev. A 86, 043819 (2012).

[5] C.W. Gardiner, P. Zoller, Quantum Noise, SpringerVerlag, Berlin 2010.

[6] R.L. Hudson, K.R. Parthasarathy, Commun. Math. Phys. 93, 301 (1984). 